\title{
Application des neutrons polarisés à l'étude des excitations magnétiques
}

\author{
L.-P. Regnault
}

CEA-Grenoble, DRFMC/SPSMS/MDN, 17 rue des Martyrs, 38054 Grenoble cedex 9, France

Dans cette partie du cours, nous présenterons les principaux aspects associés à l'utilisation de la polarimétrie en diffusion inélastique des neutrons sur des entités magnétiques. Les aspects généraux relatifs aux excitations magnétiques dans les solides et à la diffusion inélastique des neutrons seront respectivement traités dans les cours de B. Fak (CEA-Grenoble) et B. Dorner (ILL). Le plan du cours "application des neutrons polarisés a l'étude des excitations magnétiques" est le suivant:

- Généralités et définitions (neutrons polarisés et analyse de polarisation)

- La polarimétrie longitudinale: sections efficaces spin-flip et non spin-flip

- La polarimétrie sphérique

- Exemples d'utilisation des neutrons polarisés et de l'analyse de polarisation

\section{GENERALITES}

Le neutron, particule massive neutre, possède un spin 1/2 (c'est un fermion). Dans un premier temps, on va considérer un faisceau partiellement polarisé suivant la direction z. Il y a deux états possibles pour l'opérateur de spin $S_{z}$ le long de la direction de polarisation $z,+1 / 2$ et $-1 / 2$, notés respectivement $|+\rangle$ et $|-\rangle$. Si on appelle $P^{+}$la fraction de neutrons se trouvant dans l'état $|+\rangle$et $P^{-}$ la fraction de neutrons se trouvant dans l'état $|-\rangle$, on définit la polarisation du neutron par la quantité:

$$
P_{z}=P^{+}-P^{-}
$$

avec la relation supplémentaire $P^{+}+P^{-}=1$. Pour un faisceau non polarisé, on a $P^{+}=P^{-}=1 / 2$ et $P_{z}=0$. Pour un faisceau entièrement polarisé " $+", P^{+}=1$ et $P^{-}=0$ et $P_{z}=1$. Pour un faisceau entièrement polarisé "-", $P^{+}=0$ et $P^{-}=1$ et $P_{z}=-1$. Dans le cas le plus général, la polarisation du neutron est définie à partir des opérateurs de spin de Pauli $\sigma=2 S$ (§ introduction par $C$. Fermon);

$$
\begin{aligned}
& p_{x}=\left\langle\sigma_{x}\right\rangle \\
& p_{y}=\left\langle\sigma_{y}\right\rangle \\
& p_{z}=\left\langle\sigma_{z}\right\rangle
\end{aligned}
$$

avec les relations suivantes sur les vecteurs de base:

$$
\begin{aligned}
& \sigma_{x}|+\rangle=|-\rangle, \sigma_{x}|-\rangle=|+\rangle, \sigma_{y}|+\rangle=|-\rangle, \sigma_{y}|-\rangle=-i|+\rangle \\
& \text { et } \\
& \left.\sigma_{z}|+\rangle=|+\rangle, \sigma_{z}|-\rangle=-1-\right\rangle
\end{aligned}
$$


Les quatre premieres opérations qui font passer de l'état $|+\rangle$ à l'état $|-\rangle$ (et vice versa) sont dites "spin-flip". Celles qui laissent l'état de spin initial inchangé sont dites "non spin-flip". Si l'état de spin du neutron est décrit par une superposition d'état $|\sigma\rangle=a|+\rangle+b-\rangle$, on définit la polarisation $\mathbf{p}$ du neutron par les relations suivantes, déduites des relations (3):

$$
\begin{aligned}
& p_{x}=a b^{*}+a^{*} b \\
& p_{y}=i\left(a b^{*}-a^{*} b\right) \\
& p_{z}=\left.\left|d^{2}-\right| b\right|^{2}
\end{aligned}
$$

La polarisation du faisceau de neutrons, $\mathbf{P}$, est définie comme la valeur moyenne de la polarisation du neutron sur l'ensemble des neutrons constituant le faisceau:

$$
\mathbf{P}=\frac{\sum \mathbf{p}_{n}}{N}
$$

C'est, dans le cas le plus général, un vecteur (et non un opérateur). Ainsi, on peut définir la polarisation du faisceau incidente, $\mathbf{P}_{0}$, et la polarisation du faisceau après diffusion sur l'échantillon cible, $\mathbf{P}$.

La section efficace différentielle, $\frac{d^{2} \sigma}{d \Omega d \omega}$, qui caractérise finalement linteraction neutron matière dépend maintenant non seulement du transfert de moment $Q=\mathbf{k}_{1}-\mathbf{k}_{0}$ (alias vecteur de diffusion), mais aussi de la polarisation incidente $\mathbf{P}_{0}$. Les expressions générales des contributions dépendantes de $\mathbf{Q}$ de la polarisation finale $\mathbf{P}$ et de la section efficace $\frac{d^{2} \sigma}{d \Omega d \omega}$ ont été établies au début des années 60 par S. Maleyev et al et M. Blume ( $\$$ Biblio en fin de chapitre). Dans le formalisme des fonctions de corrélations, les expressions générales sont les suivantes:

$$
\begin{aligned}
& \mathbf{P} \frac{d^{2} \sigma}{d \Omega d \omega} \propto \frac{k_{1}}{k_{0}} \int_{-\infty}^{+\infty} d t e^{-i \omega t}\left\{\left\langle N_{Q}^{+} \mathbf{M}_{Q}(t)\right\rangle+\left\langle\mathbf{M}_{Q}^{+} N_{Q}(t)\right\rangle+i\left\langle\mathbf{M}_{Q} \times \mathbf{M}_{Q}^{+}(t)\right\rangle+\left\langle N_{Q} N_{Q}^{+}(t)\right\rangle \mathbf{P}_{0}+\right. \\
& \\
&\left\langle\left(\mathbf{P}_{0} \cdot \mathbf{M}_{Q}^{+}\right) \mathbf{M}_{Q}(t)\right\rangle+\left\langle\mathbf{M}_{Q}^{+}\left(\mathbf{P}_{0} \cdot \mathbf{M}_{Q}(t)\right)\right\rangle-\left\langle\mathbf{M}_{Q}^{+} \cdot \mathbf{M}_{Q}(t)\right\rangle \mathbf{P}_{0}+ \\
&\left.\left.i \mathbf{P}_{0}\left\langle\mathbf{M}_{Q}^{+} N_{Q}(t)\right\rangle-\left\langle N_{Q}^{+} \mathbf{M}_{Q}(t)\right\rangle\right]\right\}
\end{aligned}
$$

et

$$
\begin{aligned}
\frac{d^{2} \sigma}{d \Omega d \omega} \propto \frac{k_{1}}{k_{0}} \int_{-\infty}^{+\infty} d t e^{-i \omega t}\left\{N_{Q} N_{Q}^{+}(t)\right\rangle+\left\langle\mathbf{M}_{Q} \mathbf{M}_{Q}^{+}(t)\right\rangle+ & \\
& \left.\left.\mathbf{P}_{0}\left[\mathbf{M}_{Q}^{+} N_{Q}(t)\right\rangle+\left\langle N_{Q}^{+} \mathbf{M}_{Q}(t)\right\rangle-i\left\langle\mathbf{M}_{Q} \times \mathbf{M}_{Q}^{+}(t)\right\rangle\right]\right]
\end{aligned}
$$

où $N_{Q}$ et $\mathbf{M}_{Q}$ sont respectivement les opérateurs amplitude de diffusion nucléaire et amplitude de diffusion magnétique (opérateur vectoriel), donnés par les relation suivantes:

$$
\begin{aligned}
& N_{Q}=\sum_{i} b_{l} e^{Q \cdot \mathbf{R}_{l}(t)} \\
& \mathbf{M}_{\perp Q}=\mathbf{Q} \times\left[\mathbf{M}_{Q}(t) \times \mathbf{Q}\right]
\end{aligned}
$$

avec

$\mathbf{M}_{Q}=r_{0} \sum_{s} e^{i Q \cdot \mathbf{R}_{s}(t)} \mathbf{M}_{s}(t), r_{0}=\gamma e^{2} / m_{e} c^{2}=-0.54 \times 10^{-12} \mathrm{~cm}$ et $\mathbf{M}_{s}=\mathbf{S}_{s}-\frac{i}{\hbar}\left(\mathbf{Q} \times \mathbf{p}_{s}\right) / Q^{2}$

Dans ces dernières expressions, $\mathbf{R}_{t}(t)$ et $\mathbf{R}_{s}(t)$ sont les positions (dépendante du temps) des noyaux et des électrons responsables du magnétisme. 
Comme il est bien connu, $\mathbf{M}_{Q}$ met en jeu les composantes magnétiques (de spin et d'orbite) perpendiculaires au vecteur de diffusion $\mathbf{Q}$.

Les relations (6) et (7) mettent en jeux les diverses fonctions de corrélations du système. Elles mettent aussi en évidence l'existence de termes purement nucléaires $\left(\left\langle N_{Q} N_{Q}^{+}(t)\right\rangle\right)$, purement magnétiques (comme, par exemple: $\mathbf{M}_{Q} \cdot \mathbf{M}_{Q}^{+}(t)$ ou le terme chiral $i \mathbf{M}_{Q} \times \mathbf{M}_{Q}^{+}(t) \ldots$ ) et mixtes nucléaire-magnétique (comme, par exemple: $\mathbf{P}_{0}\left\lceil\left\langle\mathbf{M}_{Q}^{+} N_{Q}(t)\right\rangle \pm\left\langle N_{Q}^{+} \mathbf{M}_{Q}(t)\right\rangle\right]$ ). Ces derniers résultent en particulier de l'interférence des amplitudes de diffusion nucléaire et magnétique. Une analyse rapide des expressions ci-dessus montre que la section efficace et la polarisation contiennent des termes indépendants de la polarisation incidente $\mathbf{P}_{0}$ et des termes qui en dépendent. Dans le cas le plus général, $\mathbf{P}_{0}$ et $\mathbf{P}$ ne sont pas colinéaires: les processus d'interaction peuvent conduire à une rotation de la polarisation du faisceau différente de 0 ou $180^{\circ}$. On verra plus loin sous quelle condition. Un cas particulier important est celui pour lequel le faisceau incident n'est pas polarisé. Si dans la relation (6) on fait $\mathbf{P}_{0}=0$, on trouve pour $\mathbf{P}$ la relation suivante:

$$
\mathbf{P} \approx \frac{\int_{-\infty}^{+\infty} d t e^{-i \omega t}\left[\left\langle N_{Q}^{+} \mathbf{M}_{Q}(t)\right\rangle+\left\langle\mathbf{M}_{Q}^{+} N_{Q}(t)\right\rangle+i\left\langle\mathbf{M}_{Q} \times \mathbf{M}_{Q}^{+}(t)\right\rangle\right]}{\left.\int_{-\infty}^{+\infty} d t e^{-i \omega t}\left[N_{Q} N_{Q}^{+}(t)\right\rangle+\left\langle\mathbf{M}_{Q} \cdot \mathbf{M}_{Q}^{+}(t)\right\rangle\right]} .
$$

qui montre que le faisceau diffusé peut se polariser plus ou moins complètement suivant la force des parties réelles des termes d'interférence. C'est cette propriété qui est mise à profit dans les polariseurs à base de monocristaux ferromagnétiques (alliage d' Heusler $\mathrm{Cu}_{2} \mathrm{MnAl}$ par exemple).

Puisque la polarisation $\mathbf{P}$ est un vecteur, sa détermination complète nécessite la détermination des 3 composantes $P_{x}, P_{y}$ et $P_{z}$ (polarimétrie sphérique). Cette technique d'analyse puissante est opérationnelle en diffraction à l' $\mathrm{l} L \mathrm{~L}$ depuis une dizaine d'année (§ Tasset $e t$ al) et n'a été que très récemment mise en œuvre en diffusion inélastique des neutrons ( $\$$ Regnault, Tasset $e t$ al). Elle sera discutée plus loin en section 3. Dans la mesure où les termes d'interférences sont négligeables (ou négligés), $\mathbf{P}_{0}$ et $\mathbf{P}$ sont quasi colinéaires et la technique de polarimétrie dite "longitudinale" suffit aux études.

\section{POLARIMETRIE LONGITUDINALE}

Lors de linteraction neutron matière, il y a deux sortes de processus possibles:

ceux qui laissent inchangés l'état du spin du neutron après diffusion, associés à des processus dit "non spin-flip" (NSF):

$$
|+\rangle \rightarrow|+\rangle \text { et }|-\rangle \rightarrow|-\rangle
$$

et ceux qui changent l'état de spin du neutron, associés à des processus dit "spin-flip" (SF):

$$
|+\rangle \rightarrow \mid \rightarrow \text { et }|-\rangle \rightarrow|+\rangle
$$

On peut ainsi définir quatre sections efficaces qui dépendent des états de spin initiaux et finaux:

$\left(\frac{d^{2} \sigma}{d \Omega d \omega}\right)_{k_{0} \rightarrow k_{1}}^{++}$et $\left(\frac{d^{2} \sigma}{d \Omega d \omega}\right)_{k_{0} \rightarrow k_{1}}^{--}$, sections efficaces NSF, $\left(\frac{d^{2} \sigma}{d \Omega d \omega}\right)_{k_{0} \rightarrow k_{1}}^{+-}$et $\left(\frac{d^{2} \sigma}{d \Omega d \omega}\right)_{k_{0} \rightarrow k_{1}}^{-+}$, sections efficaces SF.

Bien entendu, on doit avoir la relation suivante reliant les sections efficaces en neutrons polarisés et non polarisés: 


$$
\left(\frac{d^{2} \sigma}{d \Omega d \omega}\right)_{k_{0} \rightarrow k_{1}}=\frac{1}{2} \times\left[\left(\frac{d^{2} \sigma}{d \Omega d \omega}\right)_{k_{0} \rightarrow k_{1}}^{++}+\left(\frac{d^{2} \sigma}{d \Omega d \omega}\right)_{k_{0} \rightarrow k_{1}}^{--}+\left(\frac{d^{2} \sigma}{d \Omega d \omega}\right)_{k_{0} \rightarrow k_{1}}^{+-}+\left(\frac{d^{2} \sigma}{d \Omega d \omega}\right)_{k_{0} \rightarrow k_{1}}^{-+}\right\rfloor
$$

Le neutron interagit avec les noyaux (diffusion "nucléaire") et avec le système électronique (diffusion "magnétique").'

\subsection{La diffusion nucléaire}

Considérons un échantillon constitué d'atomes dont le noyaux j est porteur d'un spin nucléaire $I_{j}$.

- En appelant $\left|k_{0} \sigma_{0}\right\rangle$ et $'\left|k_{1} \sigma_{1}\right\rangle$ respectivement les états initiaux et finaux du neutron, et $\left|\lambda_{0}\right\rangle$ et $\left|\lambda_{1}\right\rangle$ les états initiaux et finaux de la cible, on peut montrer que la section efficace de diffusion est donnée par la relation suivante ( $\S$ introduction et biblio):

$$
\left(\frac{d^{2} \sigma}{d \Omega d \omega}\right)^{\sigma_{0} \rightarrow \sigma_{1}}=\sum_{\lambda_{0}, \lambda_{1}} p\left(\lambda_{0}\right)\left(\frac{d^{2} \sigma}{d \Omega d \omega}\right)_{\lambda_{0} \rightarrow \lambda_{t}}^{\sigma_{0} \rightarrow \sigma_{1}}
$$

où $p(\lambda)$ est la probabilité de trouver la "cible" dans l'état $|\lambda\rangle$ et:

$$
\left(\frac{d^{2} \sigma}{d \Omega d \omega}\right)_{\lambda_{0} \rightarrow \lambda_{3}}^{\sigma_{0} \rightarrow \sigma_{1}} \propto \frac{k_{1}}{k_{0}} \mid \sum_{j}\left\langle\sigma _ { 1 } \lambda _ { 1 } | \hat { b } j e ^ { i \mathbf { Q } \cdot \mathbf { R } _ { j } } | \left\langle\left.\sigma_{0} \lambda_{0} D\right|^{2} \delta\left(E_{\lambda_{0}}-E_{\lambda_{1}}+\hbar \omega\right)\right.\right.
$$

Dans cette dernière expression, $\hat{b}_{j}$ est l'opérateur amplitude de diffusion.

Le système formé par le noyau $j$ et le neutron possède deux valeurs du spin total possible $: I_{j}+1 / 2$ et $I_{j}-1 / 2$. Pour chaque état, on peut définir une longueur de diffusion $b_{j}^{+}$et $b_{j}^{-}$qui sont respectivement les valeurs propres de l'opérateur $\hat{b}_{j}: \hat{b}_{j}|+\rangle=b_{j}^{\dagger}|+\rangle$ et $\hat{b}_{j}|-\rangle=b_{j}^{-}|-\rangle$.

Pour l'interaction nucléaire, on peut montrer que l'opérateur amplitude de diffusion est de la forme:

$$
\hat{b}_{j}=A_{j}+2 B_{j} \mathbf{I} . \boldsymbol{\sigma}
$$

avec

$$
A_{j}=\frac{1}{2 I_{j}+1}\left[\left(I_{j}+1\right) b_{j}^{+}+I_{j} b_{j}^{-}\right] \text {et } A_{j}=\frac{1}{2 I_{j}+1}\left(b_{j}^{+}-b_{j}^{-}\right)
$$

Pour l'interaction nucléaire, il y a découplage entre $\lambda$ et $\sigma$ :

$$
\left\langle\sigma _ { i } \lambda _ { 1 } | \hat { b } _ { j } e ^ { i \mathbf { Q } \cdot \mathbf { R } _ { j } } | \left\langle\sigma_{0} \lambda_{0} D=\left\langle\lambda _ { 1 } | e ^ { i \mathbf { Q } \cdot \mathbf { R } _ { j } } | \left\langle\lambda _ { 0 } D \left\langle\sigma_{1}\left|\hat{b}_{j}\right|\left\langle\sigma_{0} \mid\right\rangle\right.\right.\right.\right.\right.
$$

A partir des expressions ci dessus, on peut facilement démontrer les relations suivantes:

$$
\begin{aligned}
& \left\langle+\left|\hat{b}_{j}\right|+\right\rangle=A_{j}+B_{j} I_{z} \\
& \left\langle-\left|\hat{b}_{j}\right|-\right\rangle=A_{j}-B_{j} I_{z} \\
& \left\langle-\left|\hat{b}_{j}\right|+\right\rangle=B_{j}\left(I_{x}+i I_{y}\right) \\
& \left\langle+\left|\hat{b}_{j}\right|-\right\rangle=B_{j}\left(I_{x}-i I_{y}\right)
\end{aligned}
$$


Traditionnellement, on distingue la diffusion nucléaire cohérente et la diffusion nucléaire incohêrente.

\subsubsection{Diffusion cohérente nucléaire}

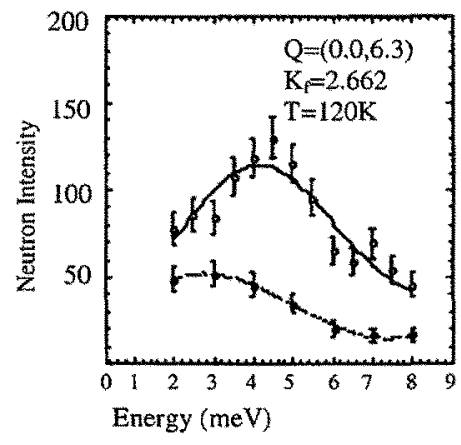

Figure 1 : Phonon acoustique dans $\mathrm{YBCO}_{6.85}$

Dans ce cas, la section efficace de diffusion inélastique dépend directement de $\bar{b}_{j}$, où la moyenne est à prendre sur les $2 I+1$ valeurs propres de l'opérateur de spin nucléaire $I_{z}$ et sur les différents isotopes de l'élément $\mathrm{j}$ :

$$
\bar{b}_{j}=\left\langle\left\langle\hat{b}_{j}\right\rangle_{\text {spin }}\right\rangle_{\text {isotope }}
$$

Compte tenu de la forme de l'opérateur $\hat{b}_{j}$, pour la moyenne sur le spin on a:

$$
\left\langle A_{j}+B_{j} I_{z}\right\rangle_{s p i n}=A_{j}+B_{j}\left\langle I_{z}\right\rangle_{s p i n}
$$

Si les spins des noyaux en question ne sont pas polarisés (ce qui est généralement le cas pour des températures supérieures à $1 \mathrm{~K}$ ), $\left\langle I_{x}\right\rangle \approx\left\langle I_{y}\right\rangle \approx\left\langle I_{z}\right\rangle \approx 0$ et on a les éléments de matrice suivant:

$$
\begin{aligned}
& \left\langle+\left|\hat{b}_{j}\right|+\right\rangle=\left\langle-\left|\hat{b}_{j}\right|-\right\rangle=\left\langle A_{j}\right\rangle_{\text {isotope }} \\
& \left\langle-\left|\hat{b}_{j}\right|+\right\rangle=\left\langle+\left|\hat{b}_{j}\right|-\right\rangle=0
\end{aligned}
$$

\section{La diffusion cohérente nucléaire est uniquement NSF.}

Ceci signifie que toutes les excitations de réseau (phonons acoustiques et optiques) ne seront détectées que dans le canal NSF: elles ne renversent pas le spin du neutron.

Exemple (figure 1): phonon dans $\mathrm{YBa}_{2} \mathrm{Cu}_{3} \mathrm{O}_{6.85}$ mesuré sur le spectromètre 3-axes CRG IN22 dans le mode $k_{f}$-constant avec $k_{f} \approx 2.662 \AA^{-1}$. Comme attendu, la contribution est essentiellement NSF, piquée autour de $4.5 \mathrm{meV}$ pour $q_{c} \approx 0.3$ r.l.u. Le canal $S F$ contient le bruit de fond "polarisé".

\subsubsection{Diffusion incohẻrente nucléaire}

Les sections efficaces dépendent maintenant de $\overline{b_{j} b_{j}^{*}}-\left(b_{j}\right)^{2}$. Le calcul des éléments de matrice se fait de façon analogue au cas cohérent. Par exemple, pour $|+\rangle \rightarrow|+\rangle$ :

Ainsi, pour la contribution nucléaire incohérente NSF, on a:

$$
\overline{b_{j} b_{j}^{*}}=\left\langle\left\langle\hat{b} \hat{b}_{j}^{+}\right\rangle_{\text {spin }}\right\rangle_{\text {isosope }}=\left\langle A_{j}^{2}+B_{j}^{2}\left\langle I_{z}^{2}\right\rangle_{\text {spin }}\right\rangle_{\text {isotope }}
$$




$$
I_{\text {incoh }}^{\text {NSF }}=\left\langle A_{j}^{2}\right\rangle_{\text {istotope }}-\left(\left\langle A_{j}\right\rangle_{\text {isotope }}\right)^{2}+\frac{1}{3}\left\langle B_{j}^{2} I_{j}\left(I_{j}+1\right)\right\rangle_{\text {isorope }}
$$

Le calcul des contributions SF $(|+\rangle \rightarrow|-\rangle)$ est similaire. On peut montrer que:

$$
I_{i n c o h}^{S F}=\frac{2}{3}\left\langle B_{j}^{2} I_{j}\left(I_{j}+1\right)\right\rangle_{\text {isotope }}
$$

L'incohérent nucléaire isotopique est uniquement NSF. L'incohérent nucléaire de spin est 1/3 NSF et 2/3 SF (facteur 2). Dans un matériau donné, la contribution SF à l'incohérent provient nécessairement du spin nucléaire alors que le NSF provient à la fois des isotopes et du spin.

Les sections efficaces ne dépendent pas de la direction de polarisation et de son orientation par rapport au vecteur de diffusion. Toutes ces propriétés ont été clairement mises en évidence sur le Vanadium, prototype du diffuseur incohérent de $\operatorname{spin}\left(\sigma_{\text {coh }} \approx 0.02\right.$ barn; $\sigma_{i n c o h} \approx 5.0$ ):

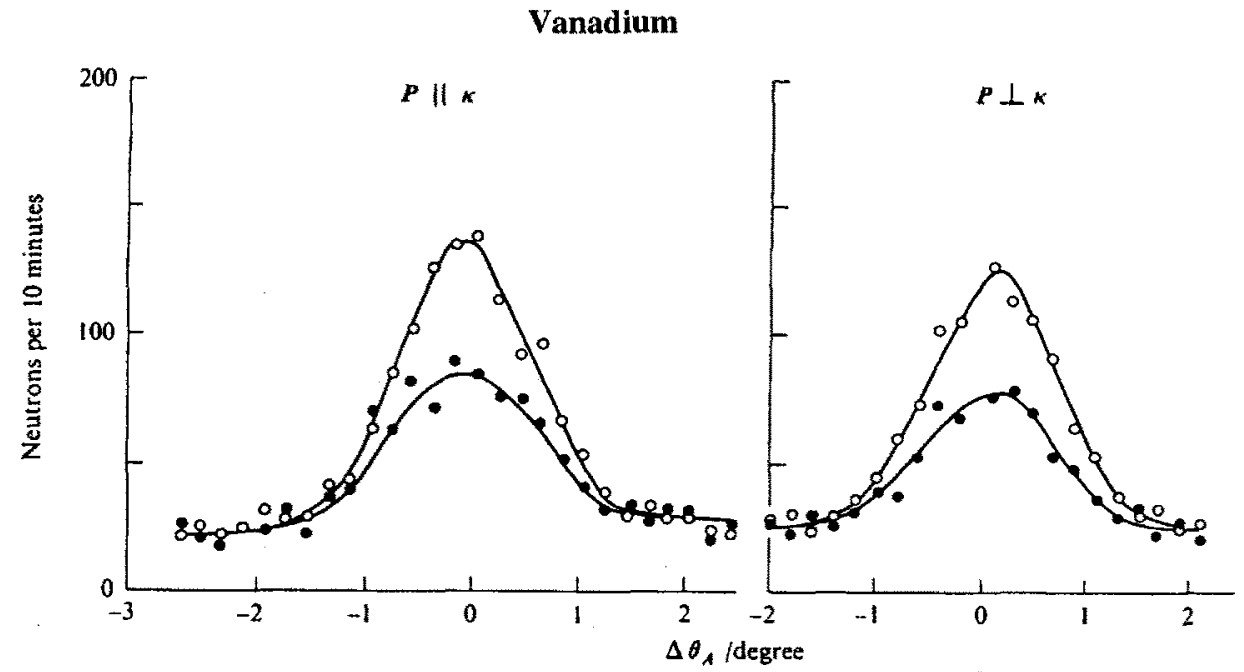

Figure 2 : échantillon de Vanadium. Les points ouverts et pleins correspondent respectivement aux contributions SF et NSF. La diffusion dans les deux cas est la même pour une polarisation incidente parallèle à $Q$ ou perpendiculaire à $Q$ (d'après Moon et al, 1969).

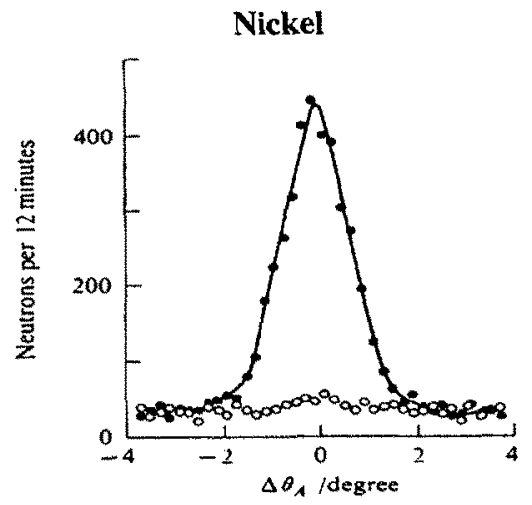

Figure 3: Diffusion incohérente du Nickel : points pleins NSF, points ouverts SF (d'après Moon et al, 1969). La diffusion incohérente isotopique est purement NSF. Un bon échantillon est le Nickel, dont tous les isotopes sont dépourvus de spin nucléaire. 


\subsection{La diffusion Magnétique}

On s'intéresse maintenant à l'interaction du neutron avec les moments magnétiques de la cible (en fait avec les électrons responsables du magnétisme de l'échantillon). Tous calculs fait ( $\$$ l'intro générale), on trouve le résultat suivant pour la section efficace (notations définies précédemment):

$$
\left(\frac{d^{2} \sigma}{d \Omega d \omega}\right)_{\lambda_{0} \rightarrow \lambda_{1}}^{\sigma_{0} \rightarrow \sigma_{1}} \propto\left(\frac{\gamma e^{2}}{m_{n} c^{2}}\right) \frac{k_{1}}{k_{0}} \mid\left.\sum_{i}\left\langle\sigma_{1} \lambda_{1}\left|\sigma \cdot \mathbf{M}_{\perp \varrho}\right|\left\langle\sigma_{0} \lambda_{0}\right|\right)\right|^{2} \delta\left(E_{\lambda_{0}}-E_{\lambda_{1}}+\hbar \omega\right)
$$

avec $\mathbf{M}_{\perp Q}=\mathbf{M}-\frac{(\mathbf{M} \cdot \mathbf{Q}) \mathbf{Q}}{Q^{2}}$, opérateur mettant en jeux les composantes de $\mathbf{M}$ perpendiculaires au vecteur de diffusion ("règle de sélection" de la diffusion magnétique des neutrons). M n'est rien d'autre que la transformée de Fourier de la densité d'aimantation $\mathbf{M}(\mathbf{R})$ dans l'échantillon. On en déduit les contributions NSF et SF de la même façon que pour la diffusion nucléaire, puisque les opérateurs $\sigma . M_{\perp Q}$ et $\sigma . I$ sont de la même forme:

$$
\begin{aligned}
& \left\langle+\left|\sigma \cdot \mathbf{M}_{\perp d}\right|+\right\rangle=M_{\perp z} \\
& \left\langle-\left|\sigma \cdot \mathbf{M}_{\perp d}\right|-\right\rangle=-M_{\perp z} \\
& \left\langle-\left|\sigma \cdot \mathbf{M}_{\perp d}\right|+\right\rangle=M_{\perp x}+i M_{\perp y} \\
& \left\langle+\left|\sigma \cdot \mathbf{M}_{\perp d}\right|-\right\rangle=M_{\perp x}-i M_{\perp y}
\end{aligned}
$$

Règle générale: Les composantes de l'aimantation parallèles au vecteur polarisation sont NSF. Les composantes de l'aimantation perpendiculaire au vecteur de polarisation sont SF. Ceci peut se voir plus simplement à partir de la précession de Larmor du spin du neutron dans le "champ" produit par l'aimantation de l'échantillon (parallèle, pas de précession, NSF; perpendiculaire, précession, SF). En diffusion inélastique, on travaille plus volontiers avec les facteurs de structures dynamiques $M^{\alpha \beta}(\mathbf{Q}, \omega)$, qui sont en fait les transformées de Fourier (TF) spatio-temporelles des fonctions de corrélation de paires:

$$
M^{\alpha \beta}(\mathbf{Q}, \omega) \stackrel{\text { TF }}{\leftrightarrow}\left\langle M_{0}^{\alpha}(0) \cdot M_{0}^{\beta}(t)\right)_{T}
$$

Le théorème "Fluctuation-dissipation" permet de relier $M^{\alpha \beta}(\mathbf{Q}, \omega)$ à la suseptibilité généralisée $\chi^{\alpha \beta}(\mathbf{Q}, \omega)$ qui caractérise le spectre des fluctuations du système (et qui est souvent la quantité calculée par les théoriciens):

$$
M^{\alpha \beta}(\mathbf{Q}, \omega) \approx \frac{\pi}{1-e^{\left(-\frac{h \omega}{k T}\right)}} \operatorname{Im}\left(\chi^{\alpha \beta}(\mathbf{Q}, \omega)\right)
$$

Pour aller plus loin, il faut connaître l'expression des facteurs de structures dynamiques et c'est là que commencent les problèmes!

Par rapport à la diffusion nucléaire, le résultat dépend maintenant de l'orientation de la direction de la polarisation incidente par rapport au vecteur de diffusion. Deux cas sont plus particulièrement intéressant: $\mathbf{P}_{0} / / \mathbf{Q}$ et $\mathbf{P}_{0} \perp \mathbf{Q}$.

Cas $\mathbf{P}_{0} \perp \mathbf{Q}$ : En supposant $\mathbf{P}_{0} / / \boldsymbol{z}$. Pour le système complet (nucléaire+magnétique) on a alors 


$$
\begin{array}{ll}
\text { NSF } & I_{\text {coh }}+I_{i n c o h}^{\text {ispotope }}+\frac{1}{3} I_{i n c o b h}^{\text {spin }}+M_{z z} \\
\text { SF } & \frac{2}{3} I_{i n c o h}^{\text {spin }}+\left(1-\frac{Q_{x}^{2}}{Q^{2}}\right) M_{x x}+\left(1-\frac{Q_{y}^{2}}{Q^{2}}\right) M_{y y}
\end{array}
$$

Cas $\mathbf{P}_{0} / / \mathbf{Q}$ : Les fluctuations parallèles à $\mathbf{Q}$ sont tuées par la règle de sélection:

$$
\begin{array}{ll}
\text { NSF } & I_{c o h}+I_{\text {incoh }}^{\text {isotope }}+\frac{1}{3} I_{i n c o h}^{s p i n}+0 \\
\text { SF } & \frac{2}{3} I_{i n c o h}^{s p i n}+\left(1-\frac{Q_{x}^{2}}{Q^{2}}\right) M_{x x}+\left(1-\frac{Q_{y}^{2}}{Q^{2}}\right) M_{y y}+M_{z z}
\end{array}
$$

Les contributions sont différentes. Mais si on considère la différence " $\perp \mathbf{Q}-/ / \mathbf{Q}$ ", on détermine directement $M_{z z}$ dans les deux canaux NSF et SF. En changeant la direction de polarisation, on peut obtenir de la même façon $M_{x x}$ et $M_{y y}$.

Ainsi, l'analyse de polarisation longitudinale permet de sortir uniquement les composantes diagonales des facteurs de structures, indépendamment du reste (séparation nucléaire/magnétisme). La méthode marche même lorsque le faisceau incident n'est pas parfaitement polarisé $\left(P_{0}<1\right)$.

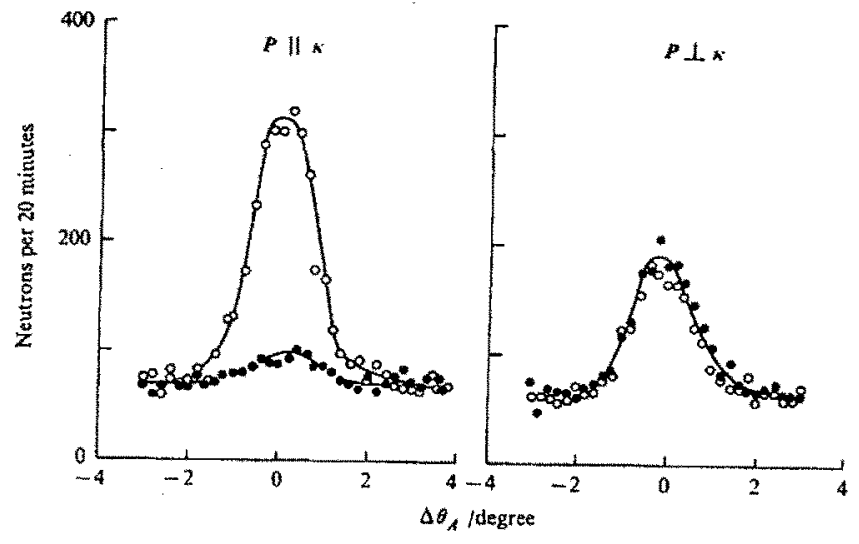

Figure 4 : Diffusion paramagnétique isotrope sur $M n F_{2}$ (d'après Moon et al, 1969)

Un exemple simple montré ci dessus: le cas de la diffusion paramagnétique isotrope. Dans ce cas, on a: $M_{x x} \approx M_{y y} \approx M_{z z} \approx M(\mathbf{Q}, \omega)$. Lorsque $\mathbf{P}_{0} \perp \mathbf{Q}$, les contributions NSF et SF sont identiques $(M(\mathbf{Q}, \omega))$. Lorsque $\mathbf{P}_{0} / / \mathbf{Q}$, la contribution NSF est nulle alors que la contribution SF vaut $2 M(\mathbf{Q}, \omega)$. Les résultats sont donc complètement différents. Ils ont été parfaitement vérifié sur le composé antiferromagnétique $\mathrm{MnF}_{2}$ (l'ion $\mathrm{Mn}^{2+}$ de spin quasi classique $\mathrm{S}=5 / 2$ est très faiblement anisotrope). En particulier le facteur 2 dans les contribution SF est quantitativement obtenu.

Pour un système ordonné, les excitations élémentaires sont les ondes de spin (alias magnons), qui correspondent à des fluctuations transverses ( $\$$ cours de B. Fak). Dans le cas où la direction des moments et la polarisation incidente sont parallèles au vecteur de diffusion, les magnons contribuent au maximum au signal SF. Dans le cas où la direction des moments et la polarisation 
incidente sont perpendiculaires à $\mathbf{Q}$ et colinéaires, il n'y a pas de contribution des magnons au signal NSF. La contribution SF sera par contre réduite par rapport au cas précédent car les fluctuations de moment parallèle à $\mathbf{Q}$ ne vont pas contribuer. Les résultats de polarimétrie longitudinale obtenus sur le système ferromagnétique $\mathrm{Li}_{0.5} \mathrm{Fe}_{2.5} \mathrm{O}_{4}$ (figure 5) mettent clairement en évidence ces prédictions.

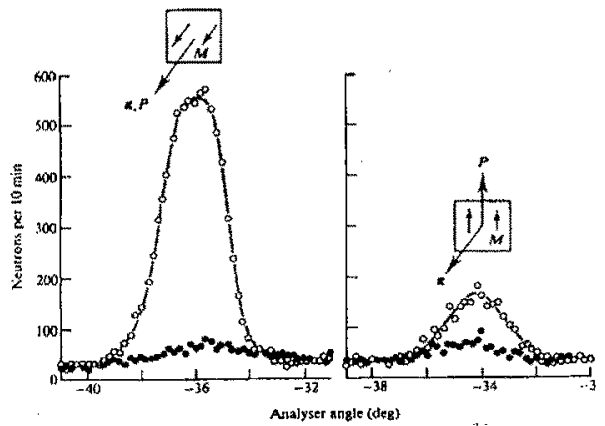

Figure 5 : polarimétrie longitudinale obtenus sur le système ferromagnétique $\mathrm{Li}_{0.5} \mathrm{Fe}_{2.5} \mathrm{O}_{4}$ (Ferrite de lithium, d'après Moon et al, 1969) points ouverts: SF; points pleins: NSF.

\subsection{La méthode expérimentale}

Le dispositif expérimental utilisé pour l'analyse de polarisation longitudinale est le suivant:

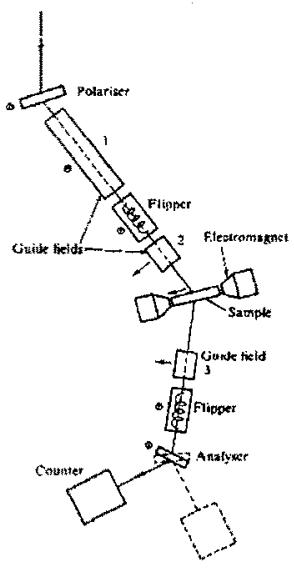

La direction de la polarisation incidente est fixée par la direction du champ de guidage appliqué sur l'échantillon. Dans la pratique, l'échantillon est plongé dans un champ magnétique de quelques dizaines de Gauss, produit par un système de bobines d'Helmholtz. La mesure des contributions SF et NSF se fait en mesurant, pour chaque configuration incidente, les contributions "flipper $O N^{\prime \prime}$ et "flipper OFF" ( $I_{O N}$ et $I_{O F F}$ ). On en déduit les contributions NSF et SF à partir des relations suivantes, où $\rho_{f}$ est le rapport de flipping:

$$
\begin{aligned}
& I_{N S F} \approx I_{O F F}-\frac{I_{O N}}{\rho_{f}} \\
& I_{S F} \approx I_{O N}-\frac{I_{O E F}}{\rho_{f}}
\end{aligned}
$$


Les polariseurs et analyseurs sur les 3-axes thermiques "modernes" sont des dispositifs à base de cristaux en alliage d'Heusler $\left(\mathrm{Cu}_{2} \mathrm{MnAl}\right)$ à géométrie focalisante. Sur les 3-axes "froids", l'étage de polarisation est très souvent assuré par un système à supermiroir associé à un monochromateur en graphite. Malgré l'efficacité des dispositifs, la perte en luminosité est énorme: de 6 (pour les neutrons froids) à prés de 15 (pour les neutrons thermiques). D'où l'obligation de temps de comptage longs (typiquement $20 \mathrm{mn}$ par point) et la nécessité de travailler à des niveaux de bruit de fond très bas (typiquement $30 \mathrm{n} /$ heure). Les rapports de flipping couramment obtenus (de l'ordre de 40 sur les 3-axes froids et de l'ordre de 20 sur les 3-axes thermiques) autorisent des déterminations précises et suffisamment découplées des contributions SF et NSF.

\section{POLARIMETRIE SPHERIQUE}

La polarimétrie longitudinale, bien que déjà puissante, ne permet cependant d'accéder qu'aux composantes diagonales du tenseur de polarisation. En particulier, la méthode décrite précédemment est incapable de faire la distinction entre une dépolarisation du faisceau réfléchit (due par exemple à l'existence de contributions magnétiques et nucléaires superposées dans l'espace réciproque) et une simple rotation de la polarisation finale. Les équations générales de la polarimétrie (6) et (7) appliquées au cas $\mathbf{P}_{0} / / \mathbf{Q}$ suggèrent néanmoins qu'une rotation de la polarisation par rapport à $-\mathbf{P}_{\mathbf{0}}$ peut exister si les termes d'interférence nucléaire-magnétique sont suffisamment forts. D'où l'intérêt des méthodes d'analyse de polarisation tridimensionnelles (alias polarimétrie sphérique), qui donnent accès avec une très grande précision aux composantes transverses de la polarisation. Dans la suite, on appellera $x$ la direction parallèle à $Q$, y la direction perpendiculaire à $Q$ dans le plan de diffusion horizontal et $\mathrm{z}$ la direction verticale. Dans le cas d'une simple rotation d'un angle $\theta$, on a les relations triviales suivantes:

$P_{x x}=-P_{0} \cos (\theta)=-P_{0} \sqrt{1-\left(\frac{P_{x y}}{P_{0}}\right)^{2}}$,

$P_{x y}=-P_{0} \sin (\theta)$

qui montrent que $P_{x x} \approx-0.95 P_{0}$ pour $P_{x y} \approx-0.32 P_{0}$ et donc toute l'ambiguitté des techniques qui ne mesurent que $P_{x x}$. Le lecteur trouvera les informations de base concernant cette nouvelle technique dans le cours de $\mathrm{F}$. Tasset consacré à la polarimétrie.

Le dispositif de base est CRYOPAD dont le schéma de principe est le suivant (d'après F. Tasset):

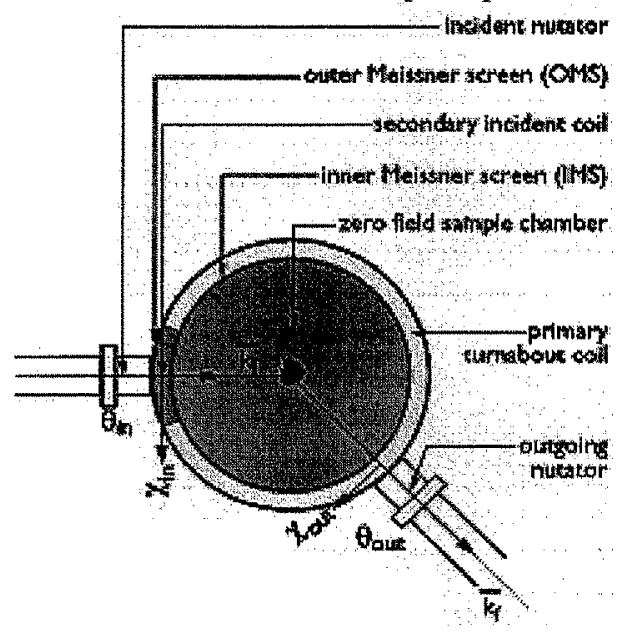


Ce dispositif expérimental permet, à partir d'un ensemble de bobinages (nutateurs et précessions) et d'écrans supraconducteur refroidis à l'hélium, de sélectionner la polarisation incidente dans les trois directions $x$, y ou $z$ et surtout, pour chaque configuration incidente, de mesurer les trois composantes de la polarisation du neutron après diffusion avec une précision de l'ordre de 0.02 . Le dispositif expérimental remplace IHelmholtz utilisé en polarimétrie longitudinale. La polarimétrie sphérique, couramment utilisée en diffraction (détermination de structures compliquées), a été appliquếe pour la première fois à la diffusion inélastique des neutrons en 1998 sur le composé $\mathrm{CuGeO}_{3}$, et depuis sur une demi douzaine de composé très différents. On donnera en section 4 des exemples de mesures sur ce composé prototype de la transition spin-Peierls.

On peut montrer, à partir des relations (6) et suivantes, que la mesure des composantes transverses de la polarisation et des termes d'interférence inélastiques donnent accès aux fonctions de corrélations "mixtes" réseau-magnétique ou magnétique-orbite. Par exemple, pour le terme $P_{x y}$ qui semble jouer un rôle privilégié, l'expression fait intervenir des fonctions de corrélations du type:

$$
\left.i \sum_{n, m} e^{i \mathbf{Q} \cdot\left(\mathbf{R}_{m}-\mathbf{R}_{n}\right)} b_{n}\left[M_{1 Q, m}(t) e^{Q \cdot\left(\mathbf{u}_{m}^{S}(t)-a_{n}^{H}(0)\right)}\right\rangle+\left\langle M_{\perp Q, m}^{+}(t) e^{-i \mathbf{Q} \cdot\left(u_{m}^{S}(t)-u_{n}^{N}(0)\right)}\right)\right]
$$

qui couplent la densité d'aimantation électronique non seulement aux positions des noyaux (spinréseau;électron-réseau), mais aussi aux positions des électrons eux-mêmes (termes orbitaux).

\section{EXEMPLES DE RESULTATS EN POLARIMETRIE INELASTIQUE}

\subsection{Séparation magnétique/nucléaire: Détermination du spectre des excitations magnétiques du supra à haute $\mathrm{Tc} \mathrm{YBa}_{2} \mathrm{Cu}_{3} \mathrm{O}_{6.85}$}

Un des points clef de la compréhension du mécanisme conduisant à l'apparition de la supraconductivité à haute température critique dans les composés de la serie $\mathrm{YBa}_{2} \mathrm{Cu}_{3} \mathrm{O}_{6+x}$ pour $x>0.4$ est la détermination précise des spectres d'excitation magnétiques et de leur variations avec la température et le dopage en "trous" (en fait les porteurs de charge) $n_{h}(x)$.

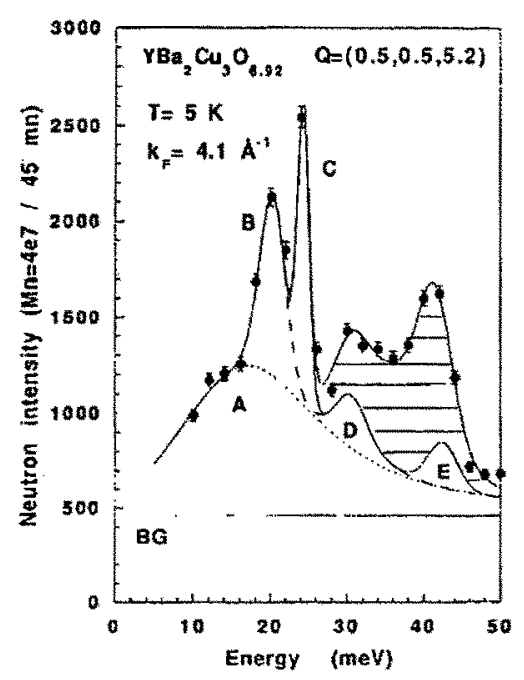

Figure 6: Diffusion inélastique sur $\mathrm{YBa}_{2} \mathrm{Cu}_{3} \mathrm{O}_{6.92}$ (neutrons non polarisés). 
Le problème a été abordé il y a une décade à partir de la diffusion inélastique des neutrons non polarisés ( $\$$ Rossat-Mignod et al.). La figure ci dessus montre un résultat typique obtenu dans le composé $\mathrm{YBa}_{2} \mathrm{Cu}_{3} \mathrm{O}_{6.92}$ qui présente de la supraconductivité à $92 \mathrm{~K}$. Clairement, les contributions magnétiques intéressantes sont "engluées" dans toute une série de contributions "parasites" (phonons, queues de Bragg, spurions non magnétiques...).

La polarimétrie longitudinale, par la mesure des contributions SF et NSF dans le cas $\mathbf{P}_{0} / / \mathbf{Q}$ (magnétisme tout SF; réseau tout NSF), permet de résoudre de façon relativement simple le problème. La figure 7 gauche montre deux scans en énergie (canal SF) obtenus aux vecteurs de diffusion $\mathbf{Q}=(3 / 2,1 / 2,1.7)$ et $(1.8,0.6,1.7)$ avec le spectromètre 3 -axes "polarisé" IN22 (CRG installé à l'ILL). Par différence, on peut avoir accès directement au spectre des excitations magnétiques. Comme il est maintenant bien admis, ce spectre révèle deux caractéristiques essentielles: une résonance autour de $40 \mathrm{meV}$ et l'existence d'un gap de spin (disparition des fluctuations magnétiques en dessous de $30 \mathrm{meV}$ ). La figure 7 (droite) montre un scan SF le long de la direction $(3,1,0)$ autour du vecteur de diffusion $(3 / 2,1 / 2,1.7)$, à une énergie de $40 \mathrm{meV}$ (c'est à dire à la résonance) obtenu sur $\mathrm{IN} 22$ (comptages de l'ordre de $20 \mathrm{mn} /$ point).
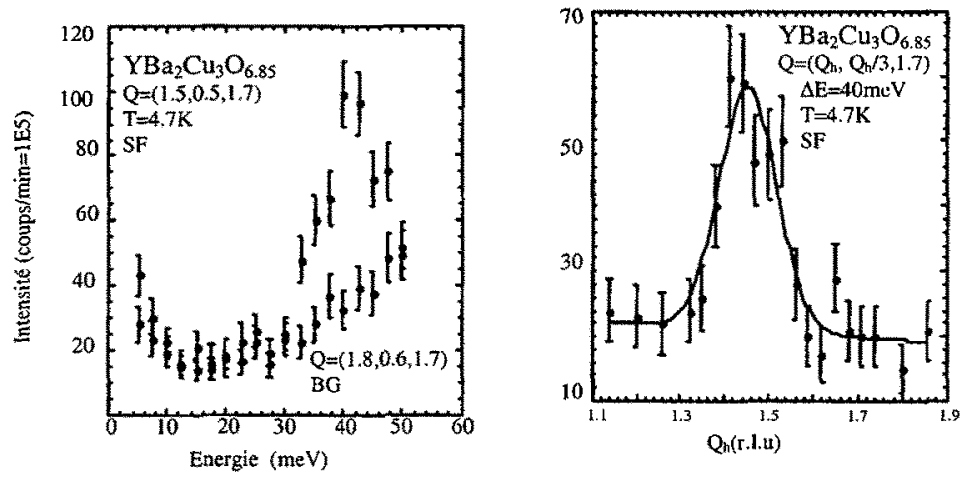

Figure 7 : Scans en énergie et en $Q$ en polarimétrie longitudinale sur $\mathrm{YBa}_{2} \mathrm{Cu}_{3} \mathrm{O}_{6.92}$.

\subsection{Détermination des différentes composantes associées aux excitations magnétiques: exemple des solitons antiferromagnétiques sous champ magnétique dans TMMC}

Les excitations magnétiques dans les chaînes antiferromagnétiques (AF) de Heisenberg à spin "classique" sont de deux sortes: les ondes de spin (excitations dites "linéaires"), qui sont associées à petits déplacements angulaires des spins et les solitons (excitations non linéaires), associés à des rotations de grande amplitude des spins. Un soliton, ce n'est en fait qu'une paroi de domaine à l'interface de deux configuations de l'état fondamental de la chaîne. A une dimension, pour des raisons topologiques évidentes, la paroi de domaine est mobile le long de la chaîne. Dans le cas antiferro, la solution qui minimise l'énergie correspond à une rotation de $180^{\circ}$ de chacun des sousréseaux. Au passage d'un soliton se produit un renversement des spins de toute une région (flipping), retoumement qui est susceptible de se voir dans la dynamique de spin. La figure 8 donne 
une vue schématique du soliton AF dans un champ magnétique appliqué perpendiculairement à la châne:

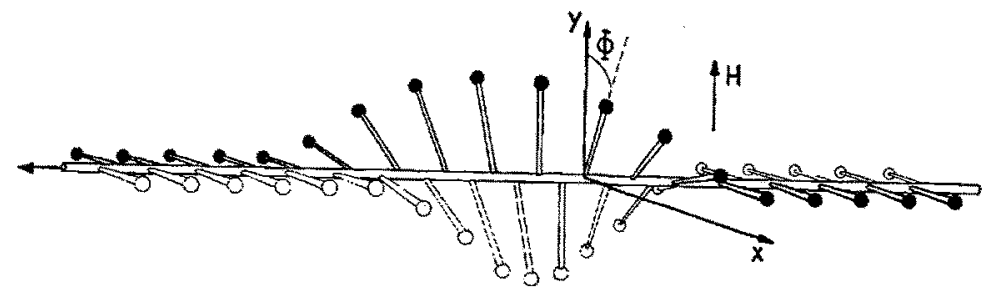

Figure 8: vue schématique d'un soliton antiferromagnétique.

L'énergie du soliton est directement reliée à la valeur du champ magnétique par la relation, $E_{s}=g \mu_{B} S H$. La distance moyenne entre deux solitons définit la longueur de corrélation du système, $\xi$, reliée à la densité de soliton $n_{s}$ par la relation $\xi^{-1}=4 n_{s}$.

Par ce qu'ils possèdent à la fois des composantes de spin parallèles et perpendiculaires au champ, les solitons vont contribuer à la fois aux facteurs de structures dynamiques $S_{J /}(q, \omega)$ et $S_{1}(q, \omega)$. En particulier, on peut vérifier que les composantes de spin du soliton lui même vont contribuer à $S_{l l}(q, \omega)$, alors que $S_{\perp}(q, \omega)$ reflètera principalement les composantes de spin inter soliton.

Les facteurs de structure dynamiques ont été calculés par H. Mikeska et K. Maki. L'allure des variations en fonction de $\mathrm{q}$ et $\omega$ est donnée sur la figure 9 ;
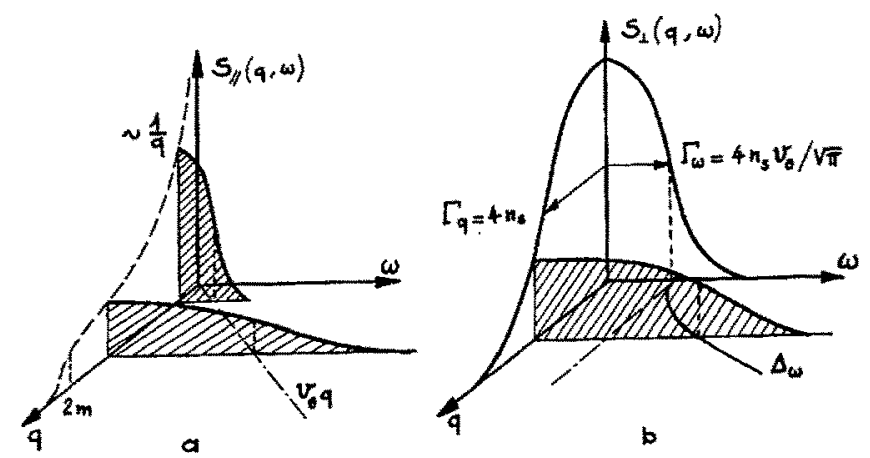

Figure 9 : facteurs de structure dynamiques calculés par H. Mikeska et K. Maki

$S_{i t}(q, \omega)$ et $S_{\perp}(q, \omega)$ sont piqués autour du point $\mathrm{AF}(" \mathrm{q}=\pi ") . S_{\perp}(q, \omega)$ a l'allure d'un "pic de Bragg" élargi à la fois en $\mathrm{q}$ et $\omega$, avec des largeurs contrôlées par la densité de soliton $\left(n_{s} \propto \frac{\left(E_{s}\right)^{3 / 2}}{T^{1 / 2}} \exp \left(-E_{s} / k T\right)\right.$, où $\mathrm{T}$ est la température). Pour des conditions habituelles $(\mathrm{T} \sim 2$ à $5 \mathrm{~K})$; $\mathrm{H} \sim 2$ à $5 \mathrm{~T}$ ), l'extension du soliton est d'au moins un ordre de grandeur plus petite que la distance entre solitons, impliquant que $S_{I /}(q, \omega) \ll S_{\perp}(q, \omega)$. 
Dans une expérience en neutrons non polarisés, $S_{\| \prime}(q, \omega)$ et $S_{\perp}(q, \omega)$ sont superposés et il est très difficile de mesurer $S_{i f}(q, \omega)$ avec précision $\left(S_{H}(q, \omega) \sim S_{\perp}(q, \omega) /(10\right.$ à 30$)$ ). Par contre, la polarimétrie longitudinale est particulièrement bien adaptée: $S_{\| \prime}(q, \omega)$, associée à des contributions parallèles au champ est entièrement NSF, alors que $S_{1}(q, \omega)$, associée à des contributions principalement perpendiculaires au champ est essentiellement SF.

Les expériences de polarimétrie longitudinales ont été effectuées sur le spectromètre 3-axes froid IN12 de l'ILL en champ allant jusqu'à $8 \mathrm{~T}\left(k_{i}\right.$ de 1.06 à $1.2 \AA^{-1}$; flipping ratio 30 ).

La figure 10 donne un exemple typique de résultat obtenu à $\mathrm{H}=4.5 \mathrm{~T}$ et $\mathrm{T}=3.6 \mathrm{~K}$.

L'analyse précise des données expérimentale montre un accord très quantitatif avec le modèle du gaz de soliton en interaction (interactions soliton-magnon et soliton-soliton).

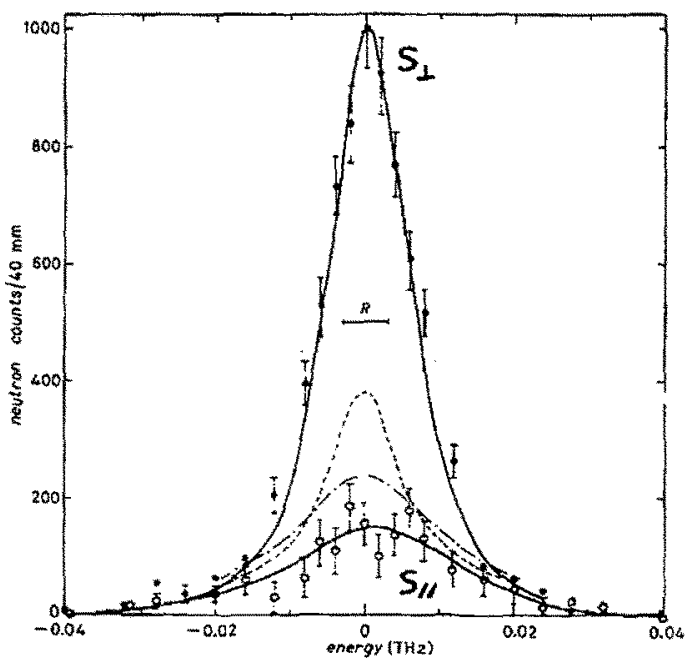

Figure 10: Frequency spectra of magnetic fluctuations observed in direction parrallel and perpendicular to the external field ( $O$ and - respectively) $(Q=(1.4,0,1.006), H=45 \mathrm{kOe}, T=3.6 \mathrm{~K})$. $R$ represents the instrumental resolution. For $S_{\perp}$, the full line is obtained from eq(10) describing the flipping mode. The theoretical curves for $S_{/ /}$are defined relative to $S_{1}$. The dashed line corresponds to non interacting soliton gas model (eq(2)). The dot-dashed line accounts for the dynamical damping of the soliton mode $(\mathrm{eq}(3))$ and the full line includes renormalization effects (eq. (9)).

\subsection{Nature "mixte" des excitations dans $\mathrm{CuGeO}_{3}$}

Le composé inorganique $\mathrm{CuGeO}_{3}$ est considéré comme un très bon exemple de système spin-Peierls pour lequel les interactions spin-réseau jouent un rôle essentiel. La chaîne uniforme antiferromagnétique de spin $1 / 2$ couplée à un champ de phonon 3D est instable et se dimérise en dessous d'une certaine température $T_{s p}$ appelée température de transition spin-Peierls. L'origine de cette instabilité résulte de la balance entre le gain d'énergie magnétique et la perte d'énergie élastique. La dimérisation, en dissymétrisant les distances entre spins proches voisins, produit une alternation de l'échange et l'ouverture d'un gap de spin d'énergie $\Delta_{s p}$ dans le spectre des excitations 
magnétiques. Les théories de champ moyen montrent que $\Delta_{s p}$ et $T_{s p}$ sont reliés par une relation de type supra-BCS: $\Delta_{s p} \approx 1.77 k T_{s p}$.

L'existence de la transition spin-Peierls et du gap de spin ont été bien mise en évidence dans $\mathrm{CuGeO}_{3}$ (§ Boucher et al). En fait, dans ce composé, la structure cristallographique comporte deux atomes par maille élémentaire le long de l'axe $\mathbf{b}$. Le spectre des excitations magnétiques se compose en fait de deux branches, symétriques par rapport au point $Q_{k}=1 / 2$ : une branche optique (d'intensités très faibles) et une branche acoustique (de l'ordre de 2 ordres de grandeur supérieurs) possédant toutes les deux un gap à $\Delta_{s p}$. Un résultat typique obtenu en neutrons polarisés (en configuration $P_{0} / / Q$ ), au vecteur de diffusion caractéristique $Q=(0,1,1 / 2)$ sur le spectromètre $C R G$ IN22 est montré figure 11 (à gauche):
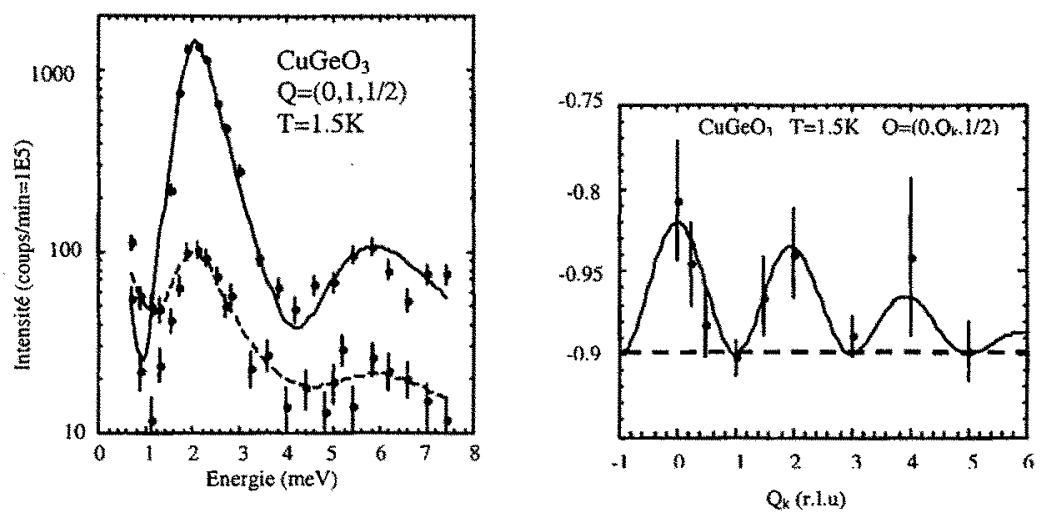

Figure 11 : Diffusion inélastique sur $\mathrm{CuGeO}_{3 .}$ Traits continus, spin flip, traits pointillés non spin flip.

En accord avec les mesures en neutrons non polarisés, le spectre des excitations magnétiques développe un gap à basse température, d'énergie $\Delta_{s p} \approx 2 \mathrm{meV}$, associé principalement à des fluctuations SF et, donc, magnétique. A ce vecteur de diffusion, la contribution résiduelle observée dans le canal NSF est entièrement attribuable à la valeur non parfaite de la polarisation incidente $\left(P_{0} \approx 0.9\right.$, impliquant un rapport de flipping fini $\approx 20$ ). Des mesures similaires à différentes composantes $Q_{k}$ montrent que la polarisation finale $\mathbf{P}$ présente un caractère "oscillant" entre les valeurs paires et impaires, qui ne peut pas s'expliquer par un effet du rapport de flipping: le faisceau diffusé subit une dépolarisation qui, suivant la discussion de la section 3 , peut se comprendre soit à partir de l'existence de terme NSF superposés, soit par un effet qui fait toumer la polarisation du neutron au-delà de $180^{\circ}$. La figure 11 (à droite) montre le résultat de la variation de la polarisation finale avec $Q_{k}$ et les oscillations associées.

Pour le mode optique, les résultats de polarimétrie longitudinale mettent en évidence des caractéristiques très différentes, comme on peut le voir sur la figure 12.

Pour $Q \approx(0,0,3 / 2)$, le mode optique présente des contributions SF et NSF a peu prés égales, signifiant que le faisceau diffusé est soit presque complètement dépolarisé, soit a presque tourné de $90^{\circ}$ par rapport à la direction $-\mathbf{P}_{0}$. Dans les deux hypothèses, cela signifie qu'une composante de 
réseau existe, superposée à la composante magnétique. Avec la polarimétrie longitudinale, il est impossible d'en dire plus sur l'amplitude de cette composante additionnelle sans modèle précis.

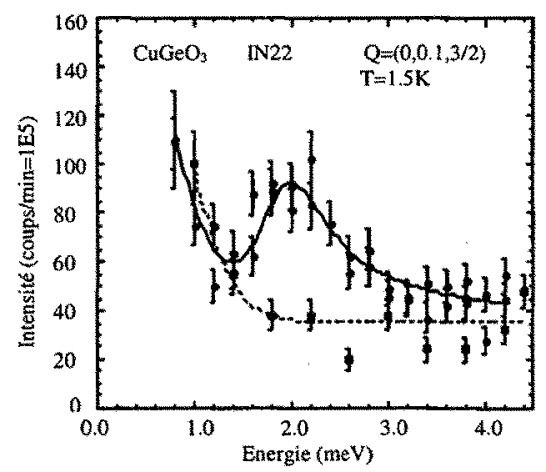

Figure 12 : mode optique de $\mathrm{CuGeO}_{3}$. Trait continu, SF. Pointillé, Bruit de fond NSF, sans traits NSF.

Cette composante nucléaire additionnelle révèle la nature "hybride" magnétique-nucléaire des excitations magnétiques dans $\mathrm{CuGeO} 3$.

\section{CONCLUSION ET PERSPECTIVES}

La polarimétrie en diffusion inélastique est une technique très puissante pour les études des spectres d'excitations magnétiques. Les neutrons polarisés et l'analyse de polarisation longitudinales conviennent pour les études de magnétisme dans la mesure où on peut négliger les termes d'interférences nucléaires-magnétiques.

Les études récentes montrent que ces termes semblent jouer un rôle certain à vecteur de diffusion "moyen", avec en particulier un effet de rotation qui vient fausser la méthode de polarimétrie longitudinale.

Seule l'analyse de polarisation "sphérique" permet de résoudre le problème de façon non ambiguë. Il est clair que, dans un avenir proche, des dispositifs du type CRYOPAD devront être utilisé de façon standard sur les spectromètres 3-axes.

\section{BIBLIOGRAPHIE}

R.M. Moon, T. Riste, et W.C. Koehler Phys. Rev. 181, 920 (1969),

M. Blume, Phys. Rev. 130, 1670 (1963.

S.V. Maleyev, V.G. Baryakhtar, et A. Suris, Sov. Phys. Solid State 4, 2533 (1963).

W. Marshall and S.W. Lovesey, dans "Theory of thermal neutron scattering", Oxford University Press (Oxford, 1971).

G.L. Squires dans "Introduction to the theory of thermal neutron scattering", Dover Pubications, Mineola, New-York, 1996.

S.W. Lovesey dans "Theory of Neutron Scattering from Condensed Matter", vol. 1 et 2, Clarendon Press, Oxford, 1987.

E. Balcar and S.W. Lovesey, dans "Theory of Magnetic Neutron and Photon Scattering", Clarendon Press, Oxford, 1989. 
F. Tasset, Physica B156-157, 627 (1989).

P.J. Brown, J.B. Forsyth, et F. Tasset, Proc. R. Soc. London A 442, 147 (1993).

L.P. Regnault, F. Tasset, T. Roberts, J.E. Lorenzo et al, Physica B 267-268, 227 (1999).

J. Rossat-Mignod et al, Physica C 185-189, 86 (1991).

L.P. Regnault, P. Bourges et P. Burlet, dans "Neutron Scattering in Layered Copper-Oxide Superconductors",ed. A. Furrer (Kluwer Academic Publishers, Dordrecht, Boston, London, 1998), p. 85 , et références incluses.

J.P. Boucher et al, Europhys. Lett. 1, 415 (1986).

H. Mikeska, J. Phys. C13, 2913, (1980).

K. Maki, J. Low. Temp. Phys. 41, 327 (1981).

J.P. Boucher et al, J. Phys. I, France, 6, 1939 (1996) et références incluses.

L.P. Regnault et J. Rossat-Mignod, dans "Magnetic properties of layered transition metal compounds", ed. L.J. de Jongh, Kluwer Academic, 1990.

\section{ANNEXE}

\subsection{Liste des spectromètres inélastiques à neutrons polarisés}

\subsubsection{Spectromètres 3-axes "froids":}

IN12: CRG ILL; Contact: S. Raymond (CEA-Grenoble); Polarimétrie longitudinale.

IN14: ILL; Contact: R. Currat (ILL), A. Wildes (ILL); Polarimétrie longitudinale option polarimétrie sphérique disponible fin 2001.

4F1: LLB; Contact: M. Hennion (LLB), P. Bourges (LLB); Polarimétrie longitudinale.

\subsubsection{Spectromètres 3-axes "thermiques":}

IN20: ILL; Contact: R. Currat (ILL), J. Kulda (ILL); Polarimétrie longitudinale et sphérique. IN22: CRG ILL; Contact: L.P. Regnault (CEA-Grenoble); Polarimétrie longitudinale et option polarimétrie sphérique disponible fin 2000.

2T: LLB; Contact: P. Bourges (LLB); Option polarimétrie longitudinale disponible fin 2000. 